\title{
Economic policy uncertainty and stock market volatility
}

\author{
$\operatorname{Li~Liu~}^{\mathrm{a}}$, Tao Zhang ${ }^{\mathrm{b}} *$ \\ ${ }^{a}$ School of Finance, Nanjing Audit University \\ ${ }^{\mathrm{b}}$ School of Economics and Management, China University of Petroleum (East China), \\ Qingdao, China \\ *Corresponding author \\ Email: liuli840821@nau.edu.cn (Li Liu), zht2129@126.com (Tao Zhang) \\ Corresponding address: Changjiang West Rd.66, Economic \& Technological \\ Development Zone, Qingdao, Shandong, PR China \\ Corresponding Tel: +86 18954250671
}

\begin{abstract}
This paper investigates the predictability of economic policy uncertainty (EPU) to stock market volatility. Our in-sample evidence suggests that higher EPU leads to significant increases in market volatility. Out-of-sample findings show that incorporating EPU as an additional predictive variable into the existing volatility prediction models significantly improves forecasting ability of these models. The improvement is robust to the model specifications.

Keywords: Economic policy uncertainty; Realized volatility; Predictability JEL codes: C53; E44; E52; E60
\end{abstract}




\section{Introduction}

Economic policy uncertainty (EPU) has been the subject of extensive investigation since the EPU index was constructed by Baker et al. (2013) (see, e.g., Antonakakis et al., 2013; Colombo, 2013; Karnizova and Li, 2014; Klößner and Sekkel, 2014; Wang et al., 2015). Notably, there are many empirical papers that investigate the effects of EPU on stock market return or volatility (see, e.g., Amengual and Xiu, 2015; Antonakakis et al., 2013; Balcilar et al., 2013; Brogaard and Detzel, 2015; Fanta and Sum, 2013; Johnson and Lee, 2013; Kang and Ratti, 2012; Lam and Zhang, 2013). In this study, we contribute to the literature by examining predictability of EPU to stock market volatility. In particular, we investigate whether adding EPU to the existing volatility prediction models can improve forecasting ability. This issue has not been addressed in the existing studies. Moreover, we show out-of-sample results whereas existing studies focus on in-sample relationships between EPU and volatility only. Therefore, our study provides additional financial implications because out-of-sample volatility forecasts are of great importance for portfolio allocation and financial risk management.

We use the 5-min high-frequency return of the S\&P 500 to calculate daily realized market volatility, and eight popular models that capture evolution of daily realized volatility $(\mathrm{RV})$ to examine the role of EPU. Our in-sample evidence indicates that the effects of the past EPU on the current RV are significantly positive, regardless of which model is used. This is consistent with the finding in Antonakakis et al. (2013) that a rise in policy uncertainty increases stock market return uncertainty. 
Out-of-sample results also suggest that EPU plays a significant role in predicting market realized volatility. Overall, we find that EPU helps to predict market volatility both in-sample and out-of-sample. Our finding is intrinsically consistent with the work of Karnizova and Li (2014), which indicates that EPU indexes help predict economic recessions. Business-cycle fluctuation is one of the most important driving forces of stock market activity (Rapach and Zhou, 2013). The predictability of EPU to stock volatility can be explained by a general equilibrium model developed by Pastor and Veronesi (2012), hereafter PV. PV find that introducing new policies with an uncertain impact increases the volatility of the stochastic discount factor. The increase in the volatility of the discount factor leads to increases in risk premia which in turn result in high volatility in stock market.

The remainder of this paper is organized as follows. Section 2 briefly describes the realized volatility models. Section 3 presents the data and discusses the main empirical results. Section 4 presents our conclusions.

\section{Methodology}

\subsection{Realized volatility}

Andersen and Bollerslev (1998)'s pioneering study proposes using realized volatility (RV) as a proxy for integrated variance. For a specific business day $t$, the realized volatility can be calculated as the sum of the squared intraday returns $r_{t, j}$ :

$$
R V_{t}=\sum_{j=1}^{M} r_{t, j}^{2}, t=1,2, \ldots T
$$

where $1 / M$ is the given sampling frequency. As we use 5-min intraday data, we have $M=48$. 


\subsection{Volatility models}

The goal of this study is to examine the ability of EPU to predict the realized volatility (RV). As the results may depend on the model specification, we use the following the eight popular volatility models rather than make a single choice.

We first use the heterogeneous autoregressive RV (HAR-RV) of Corsi et al. (2009). This model is simple to implement, as it only contains three explanatory variables: lagged daily realized volatility $\left(R V_{d, t}\right)$, lagged weekly realized volatility $\left(R V_{w, t}\right)$ and lagged monthly realized volatility $\left(R V_{m, t}\right)$. To examine the ability of EPU to predict RV, we add the lagged daily EPU to the volatility equation as a predictor. A modified HAR with EPU can then be written as follows:

$$
R V_{t+1}=\beta_{0}+\beta_{d} R V_{t}+\beta_{w} R V_{w, t}+\beta_{m} R V_{m, t}+\theta E P U_{t}+\varepsilon_{t+1},
$$

where $R V_{w, t}$ is the average $\mathrm{RV}$ from day $\mathrm{t}-5$ to day $\mathrm{t}-1$, and $R V_{m, t}$ is the average $\mathrm{RV}$ from day $\mathrm{t}-22$ to $\mathrm{t}-1$.

Andersen et al. (2007) extend HAR-RV by adding the jump component and introduce the HAR-RV-J model accordingly. The specification of HAR-RV-J with EPU is

$$
R V_{t+1}=\beta_{0}+\beta_{d} R V_{t}+\beta_{w} R V_{w, t}+\beta_{m} R V_{m, t}+\beta_{j, d} J_{t}+\theta E P U_{t}+\varepsilon_{t+1},
$$

where $J_{t}=\max \left(\mathrm{RV}_{t}-\mathrm{BPV}_{t}, 0\right), \quad B P V_{t}=u_{1}^{-2} \sum_{j=2}^{M}\left|r_{t, j-1}\right|\left|r_{t, j}\right|$ and $u_{1}=(2 / \pi)^{0.5}=E(|Z|) \quad$ is the mean of the absolute value of the standard normally distributed random variable $Z$.

Andersen et al. (2007) further decompose RV into a continuous sample path and jumps. Based on this decomposition, the following HAR-RV-CJ model is proposed:

$$
R V_{t+1}=\beta_{0}+\beta_{c, d} C S P_{t}+\beta_{c, w} C S P_{w, t}+\beta_{c, m} C S P_{m, t}+\beta_{j, d} C J_{t}+\beta_{j, w} C J_{w, t}+\beta_{j, m} C J_{m, t}+\theta E P U_{t}+\varepsilon_{t+1},(4)
$$


where $C J_{t}=I\left(Z_{t}>\Phi_{\alpha}\right) \cdot\left[R V_{t}-B P V_{t}\right], \quad C S P_{t}=I\left(Z_{t} \leq \Phi_{\alpha}\right) \cdot R V_{t}+I\left(Z_{t}>\Phi_{\alpha}\right) \cdot B P V_{t}$ and $I(\cdot)$ is the indicator function. $\operatorname{CSP}_{w, t}\left(C J_{w, t}\right)$ and $\operatorname{CSP}_{m, t}\left(C J_{m, t}\right)$ are the weekly and monthly averages of the continuous sample path and jump, respectively.

Corsi et al. (2010) modify the jump statistic in Equation (4) and propose following the HAR-RV-TCJ model accordingly:

$$
R V_{t+1}=\beta_{0}+\beta_{c, d} T C_{t}+\beta_{c, w} T C_{w, t}+\beta_{c, m} T C_{m, t}+\beta_{j, d} T J_{t}+\theta E P U_{t}+\varepsilon_{t}
$$

The aforementioned volatility models do not capture the role of the "leverage effect" in volatility dynamics. For this reason, Patton and Sheppard (2013) develop a series of models using signed realized measures. The first model is the extension of the standard HAR-RV by decomposing the daily RV into two semi-variances (HAR-RV-RS-I ),

$$
R V_{t+1}=\beta_{0}+\beta_{d}^{+} R S_{t}^{+}+\beta_{d}^{-} R S_{t}^{-}+\beta_{w} R V_{w, t}+\beta_{m} R V_{m, t}+\theta E P U_{t}+\varepsilon_{t+1},
$$

where $R S_{t}^{-}=\sum_{j=1}^{M} r_{t, j}^{2} I\left(r_{t, j}<0\right)$ and $R S_{t}^{+}=\sum_{j=1}^{M} r_{t, j}^{2} I\left(r_{t, j}>0\right)$

The second model (HAR-RV-RS-II) adds a term that interacts the lagged realized variance with an indicator for negative daily returns $R V_{t} I\left(r_{t}<0\right)$. The equation of this model is

$$
R V_{t+1}=\beta_{0}+\beta_{d}^{+} R S_{t}^{+}+\beta_{d}^{-} R S_{t}^{-}+\gamma R V_{d, t} I\left(r_{t}<0\right)+\beta_{w} R V_{w, t}+\beta_{m} R V_{m, t}+\theta E P U_{t}+\varepsilon_{t+1},(7)
$$

The third model for capturing the "leverage effect" contains the signed jump variation and an estimator of the variation due to the continuous part (bipower variation) (HAR-RV-SJ-I):

$$
R V_{t+1}=\beta_{0}+\beta_{j, d} S J_{t}+\beta_{b v, d} B V_{t}+\beta_{w} R V_{w, t}+\beta_{m} R V_{m, t}+\theta E P U_{t}+\varepsilon_{t+1},
$$

where $S J_{t}=R S_{t}^{+}-R S_{t}^{-}$. 
The final model for "leverage effect" disentangles the role of positive and negative jumps, respectively (HAR-RV-SJ-II ):

$$
R V_{t+1}=\beta_{0}+\beta_{j, d}^{+} S J_{t}^{+}+\beta_{j, d}^{-} S J_{t}^{-}+\beta_{b v, d} B V_{t}+\beta_{w} R V_{w, t}+\beta_{m} R V_{m, t}+\theta E P U_{t}+\varepsilon_{t+1}
$$

where $S J_{t}^{+}=S J_{t} \mathrm{I}\left(S J_{t}>0\right)$ and $S J_{t}^{-}=S J_{t} \mathrm{I}\left(S J_{t}<0\right)$.

Overall, we use a total of eight HAR-type volatility equations to model and forecast the realized volatility.

\section{Data and Empirical Results}

\subsection{Data description and summary statistics}

We use 5-min high frequency data from the Standard and Poor's 500 (S\&P 500) index collected from Datastream. Our sample data are for the trading time in each business day between 9:30:00 and 16:00:00 and cover the period from January 2, 1996 to June 24, 2013. After removing the data for days with too few transactions, we finally obtain 4280 trading days. We calculate RV using percent returns of closing prices. Baker et al.'s (2013) index of EPU is downloaded from the Economic Policy Uncertainty website (http://www.policyuncertainty.com/). We use the EPU index divided by 100 in empirical analysis. The summary statistics of RV and EPU are given in Table 1.

\section{Insert Table 1 here}

\subsection{The marginal effects of EPU}

Table 2 reports the in-sample estimation results of the eight volatility models with the additional predictor of EPU. We can see that the parameter of EPU is significantly positive at the $5 \%$ level, regardless of the volatility model that is used. 
The regression coefficients are also relatively stable when the volatility model changes. This finding suggests the strong ability of EPU to predict RV. We calculate the effects of one standard deviation increase in policy uncertainty in terms the percent change in volatility, as well as the two standard error upper and lower bands, using the regression coefficients of EPU. The results in Table 3 suggest that the marginal effect of EPU is about 0.03-0.04 percent, depending on the model specification. The significant impacts of EPU are consistent with the findings in recent studies that macroeconomic news can affect stock volatility (see, e.g., Asgharian et al., 2015; Onan et al., 2014).

\section{Insert Table 2 and Table 3 here}

Stock market volatility is of course an important source of economic uncertainty. Therefore, it may be argued that the significant marginal effect of EPU is caused by the fact that policy uncertainty variable and S\&P volatility are endogenous. Actually, a larger fraction of policy uncertainty is due to the uncertainty about policy-related macroeconomic variables such as Consumer Price Index, Government Budget and Federal Expenditure. These variables are exogenous to stock market volatility at least in the short-term. Another considerable part of policy uncertainty comes from events that are exogenous to stock market volatility such as Gulf Wars and the Clinton and Obama Election. From above analysis, we can conclude that our policy uncertainty variable is exogenous to $\mathrm{S} \& \mathrm{P}$ volatility to a large extent. Baker et al.(2013) also argue that their policy uncertainty index have substantial independent variation to VIX.

\subsection{Is EPU helpful for predicting RV?}


Compared with the in-sample results, market participants may be more concerned about out-of-sample performance because they would like to know how well they can use the model in the future. For this reason, we examine whether adding EPU to a volatility model can improve its predictive ability for out-of-sample data.

We use the rolling window method in our forecasting procedure. Specifically, we divide the whole sample data into two subsamples: in-sample data covering the first 2000 trading days for parameter estimation, and out-of-sample data covering the remaining 2280 days for evaluating the forecasting performance. The estimation period is then rolled forward by adding a new day and dropping the most distant day. In this way, the sample size employed to estimate the model parameters is fixed, and we re-estimate the parameters of these models each day to obtain volatility forecasts.

We obtain volatility forecasts for the horizons of 1 day and 20 days. To measure the forecasting accuracy, we use the following two heteroscedasticity-adjusted loss functions suggested by Bollerslev and Ghysels (1996):

$$
\begin{aligned}
& \mathrm{HMSE}=n^{-1} \sum_{t=1}^{n}\left(1-R V_{t} / h_{t}\right)^{2}, \\
& \mathrm{HMAE}=n^{-1} \sum_{t=1}^{n}\left|1-R V_{t} / h_{t}\right|,
\end{aligned}
$$

where $n$ is the number of volatility forecasts $(n=2280), R V_{t}$ is the actual realized volatility and $h_{t}$ is the volatility forecast. In addition, we use the Diebold and Mariano (1995) (DM) test to examine whether the differences in the loss functions between the two models are significant.

We compare the forecasting accuracy between the volatility model with EPU and 
that without EPU and display the results in Table 4. For a forecasting horizon of 1 day, the forecasting loss of a model with EPU is smaller than that without EPU, regardless of the model employed. Moreover, the p-values of the DM test indicate that after adding EPU as one more predictor, the improvement in predictive ability is significant at the $5 \%$ level for each of eight volatility models. For the 20-day horizon, the gains in predictability are relatively weak but still significant at the $10 \%$ level. This result suggests that EPU has the ability to predict RV for out-of-sample data.

\section{Insert Table 4 here}

\section{Concluding Remarks}

In a recent paper, Pastor and Veronesi (2012) explain theoretically how policy uncertainty affects stock market volatility. This paper improves on their work by empirically examining whether economic policy uncertainty (EPU) can predict future market volatility. Our in-sample as well as out-of-sample evidence suggests that EPU exhibits significantly predictive power of market volatility which is robust to the specifications of the volatility prediction models considered. Overall, our findings indicate that EPU has predictive ability to stock market volatility.

\section{Acknowledgements}

We would like to show our sincere gratitude to the chief editor (Douglas Cumming) and an anonymous referee whose comments and suggestions greatly improved the quality of this paper. This study is supported by the National Science Foundation of China (No.71401077). 


\section{References}

Amengual, D., Xiu, D., 2014. Resolution of policy uncertainty and sudden declines in volatility. Chicago Booth Research Paper, (13-78).

Andersen, T.G., Bollerslev, T., 1998. Answering the skeptics: yes, standard volatility models do provide accurate forecasts. International Economic Review 39, $885-905$.

Andersen, T.G., Bollerslev, T., Diebold, F.X., 2007. Roughing it up: Including jump components in the measurement, modeling, and forecasting of return volatility. The Review of Economics and Statistics 89, 701-720.

Antonakakis, N., Chatziantoniou, I., Filis, G., 2013. Dynamic co-movements of stock market returns, implied volatility and policy uncertainty. Economics Letters 120, $87-92$.

Asgharian, H., Christiansen, C., Hou, A. J., 2015. Effects of macroeconomic uncertainty on the stock and bond markets. Finance Research Letters 13, 10-16.

Baker, S.R., Bloom, N., Davis, S.J., 2013. Measuring economic policy uncertainty. Working paper, Stanford University.

Balcilar, M., Chang, T., Gupta, R., Li, X., 2013. The causal relationship between economic policy uncertainty and stock returns in China and India: evidence from a bootstrap rolling-window approach. Working paper. University of Pretoria.

Brogaard, J., Detzel, A., 2015. The asset-pricing implications of government economic policy uncertainty. Management Science 61, 3-18.

Bollerslev, T., Ghysels, E., 1996.. Periodic autoregressive conditional 
heteroscedasticity. Journal of Business \& Economic Statistics 14, 139-151.

Colombo, V., 2013. Economic policy uncertainty in the US: Does it matter for the Euro area?. Economics Letters 121, 39-42.

Corsi, F., 2009. A simple approximate long-memory model of realized volatility. Journal of Financial Econometrics 159, 276-288.

Corsi, F., Reno, R., 2012. Discrete-time volatility forecasting with persistent leverage effect and the link with continuous-time volatility modeling. Journal of Business and Economic Statistics 30, 368-380.

Diebold, F. X., Mariano, R.S., 1995. Comparing predictive accuracy. Journal of Business and Economic Statistics 13, 253-263.

Fanta, F., Sum, V., 2012. Long-Run Relation and Speed of Adjustment of Economic Policy Uncertainty and Excess Return Volatility. International Research Journal of Finance and Economics 102, 6-12.

Johnson, T. C., Lee, J., 2014. On the systematic volatility of unpriced earnings. Journal of Financial Economics 114, 84-104.

Kang, W., Ratti, R. A., 2013. Oil shocks, policy uncertainty and stock market return. Journal of International Financial Markets, Institutions and Money 26, 305-318.

Karnizova, L., Li, J., 2014. Economic policy uncertainty, financial markets and probability of US recessions. Economics Letters 125, 261-265.

Klößner, S., Sekkel, R., 2014. International spillover of policy uncertainty. Economics Letters 124, 508-512. 
Lam, S. S., Zhang, W., 2014. Does Policy Uncertainty Matter for International Equity Markets?. Available at SSRN 2297133.

Onan, M., Salih, A., Yasar, B., 2014. Impact of macroeconomic announcements on implied volatility slope of SPX options and VIX. Finance Research Letters 11, 454-462.

Pastor, L., Veronesi, P., 2012. Uncertainty about government policy and stock prices. Journal of Finance 67, 1219-1264.

Patton, A.J., Sheppard, K., 2013. Good volatility, bad volatility: Signed jumps and the persistence of volatility. Working paper, Duke University.

Rapach, D., Zhou, G., 2013. Forecasting stock returns. In Handbook of Forecasting II, edited by G. Elliott and A. Timmerman. North-Holland.

Wang, Y., Zhang, B., Diao, X., Wu, C., 2015. Commodity price changes and the predictability of economic policy uncertainty. Economics Letters 127, 39-42.

\section{Tables}

Table 1 Descriptive statistics of Realized volatility and policy uncertainty index

\begin{tabular}{ccccccc}
\hline & Mean & Max. & Min. & Std.Dev. & Skewness & Kurtosis \\
\hline RV & 1.035 & 18.62 & 0.046 & 1.480 & 5.033 & 35.78 \\
EPU & 0.982 & 7.191 & 0.047 & 0.706 & 2.019 & 7.477 \\
\hline
\end{tabular}

Notes: RV is the realized volatility calculated for the percent returns. EPU is the economic policy uncertainty index divided by 100 . 
Table 2 Estimation results of volatility models

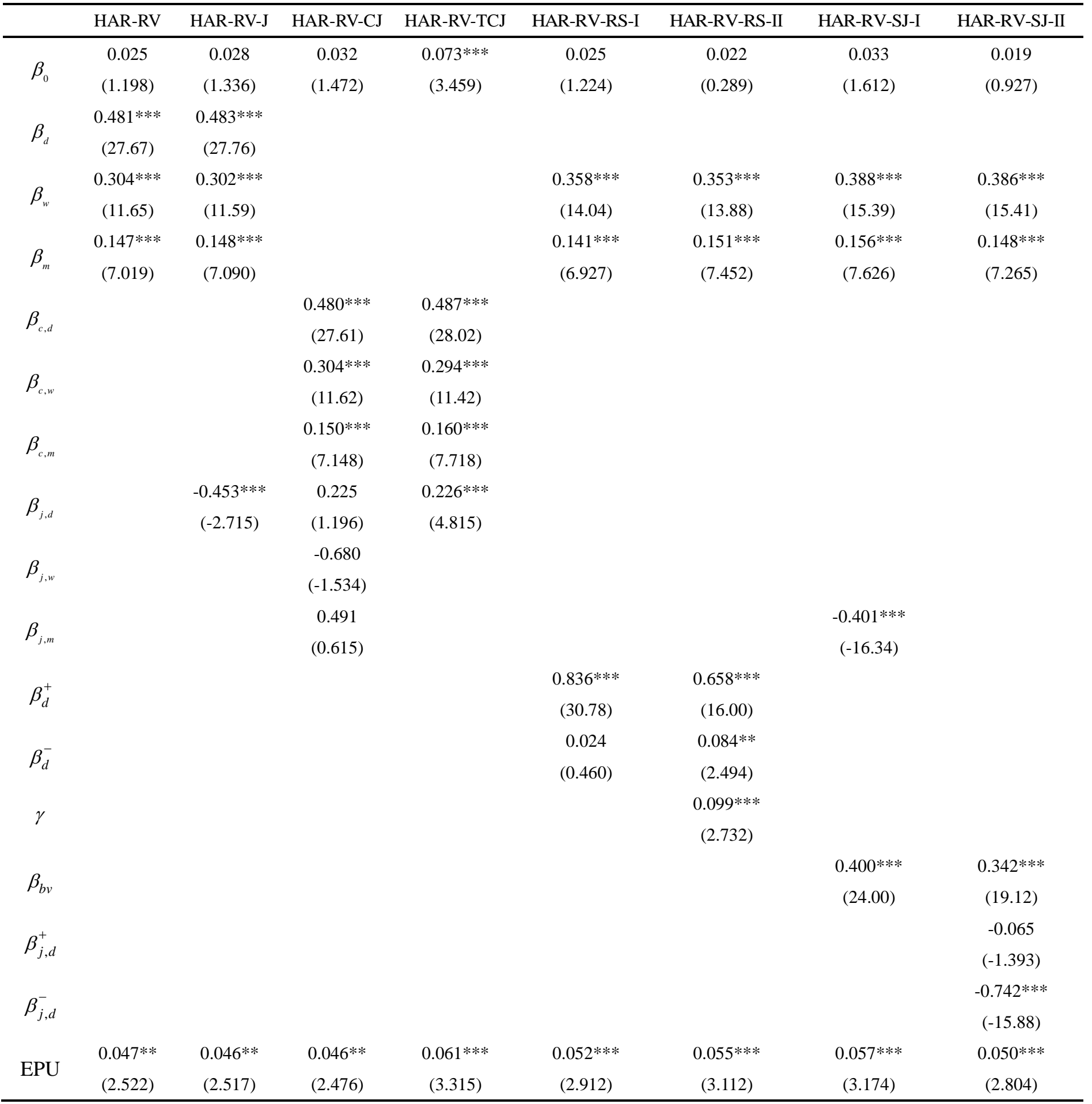

Notes: This table provides the parameter estimation results for the realized volatility models. The numbers in the parentheses are the t-statistics. The asterisks $* * *$ and $* * *$ denote rejections of null hypothesis at $10 \%, 5 \%$ and $1 \%$ significance levels, respectively. 
Table 3 Marginal effect of economic policy uncertainty on daily volatility

\begin{tabular}{lcccccccc}
\hline & HAR-RV & HAR-RV-J & HAR-RV-CJ & HAR-RV-TCJ & HAR-RV-RS-I & HAR-RV-RS-II & HAR-RV-SJ-I & HAR-RV-SJ-II \\
\hline Average effect & 0.033 & 0.032 & 0.032 & 0.043 & 0.037 & 0.039 & 0.040 & 0.035 \\
Upper band & 0.060 & 0.058 & 0.059 & 0.069 & 0.062 & 0.064 & 0.066 & 0.061 \\
Lower band & 0.007 & 0.007 & 0.006 & 0.017 & 0.012 & 0.014 & 0.015 & 0.010 \\
\hline
\end{tabular}

Notes: This table provides the effects of one standard deviation increase in policy uncertainty index in terms of the percentage change in volatility. We also give the upper and lower bands of two standard errors.

Table 4 Forecasting results of volatility models

\begin{tabular}{|c|c|c|c|c|c|c|}
\hline \multirow[b]{2}{*}{ Horizon=1 } & \multicolumn{3}{|c|}{ HMSE } & \multicolumn{3}{|c|}{ HMAE } \\
\hline & & & & & & \\
\hline & $\begin{array}{l}\text { Model with } \\
\text { EPU }\end{array}$ & $\begin{array}{c}\text { Model } \\
\text { without EPU }\end{array}$ & $\begin{array}{c}\text { p-values of } \\
\text { DM statistics }\end{array}$ & $\begin{array}{l}\text { Model with } \\
\text { EPU }\end{array}$ & $\begin{array}{c}\text { Model } \\
\text { without EPU }\end{array}$ & $\begin{array}{c}\text { p-values of } \\
\text { DM statistics }\end{array}$ \\
\hline HAR-RV & 0.346 & 0.373 & 0.007 & 0.413 & 0.418 & 0.001 \\
\hline HAR-RV-J & 0.345 & 0.370 & 0.007 & 0.412 & 0.418 & 0.001 \\
\hline HAR-RV-CJ & 0.343 & 0.367 & 0.008 & 0.412 & 0.417 & 0.001 \\
\hline HAR-RV-TCJ & 0.331 & 0.356 & 0.011 & 0.411 & 0.417 & 0.001 \\
\hline HAR-RV-RS-I & 0.337 & 0.366 & 0.007 & 0.403 & 0.407 & 0.007 \\
\hline HAR-RV-RS-II & 0.338 & 0.366 & 0.008 & 0.403 & 0.408 & 0.006 \\
\hline HAR-RV-SJ-I & 0.327 & 0.354 & 0.013 & 0.401 & 0.406 & 0.016 \\
\hline HAR-RV-SJ-II & 0.356 & 0.388 & 0.013 & 0.410 & 0.416 & 0.001 \\
\hline \multicolumn{7}{|l|}{ Horizon $=20$} \\
\hline HAR-RV & 1.248 & 1.337 & 0.053 & 0.658 & 0.663 & 0.074 \\
\hline HAR-RV-J & 1.265 & 1.355 & 0.052 & 0.659 & 0.665 & 0.056 \\
\hline HAR-RV-CJ & 1.371 & 1.453 & 0.073 & 0.664 & 0.670 & 0.055 \\
\hline HAR-RV-TCJ & 1.294 & 1.437 & 0.035 & 0.659 & 0.667 & 0.056 \\
\hline HAR-RV-RS-I & 1.244 & 1.322 & 0.065 & 0.658 & 0.663 & 0.073 \\
\hline HAR-RV-RS-II & 1.211 & 1.299 & 0.055 & 0.656 & 0.662 & 0.052 \\
\hline HAR-RV-SJ-I & 1.238 & 1.318 & 0.063 & 0.657 & 0.663 & 0.075 \\
\hline HAR-RV-SJ-II & 1.242 & 1.327 & 0.055 & 0.657 & 0.663 & 0.063 \\
\hline
\end{tabular}

Notes: This table provides the loss functions of different volatility models with economic policy uncertainty (EPU) and those without EPU. The p-values of Diebold and Mariano (1995) test are also given. 